\title{
Metal Enrichment of the Intra-Cluster Medium: Ram-Pressure Stripping of Cluster Galaxies and the Feedback of Intra-Cluster Supernovae
}

\author{
Wilfried Domainko* \\ Inst. for Astroph., Innsbruck, Austria \\ E-mail: wilfried.domainko@uibk.ac.at
}

\author{
W. Kapferer, M. Gitti, S. Schindler, E. van Kampen, S. Kimeswenger, M. Mair, T. \\ Kronberger \\ Inst. for Astroph., Innsbruck, Austria
}

\section{Ruffert, O. E. Mangete}

Department of Mathemaics and Statisitics, Univerity of Edinburgh, UK

\begin{abstract}
The Intra-Cluster Medium (ICM) contains a significant amount of metals. As heavy elements are produced in stars there are two principle ways to enrich the ICM. On the one hand chemical enriched material is produced by the stellar population of the cluster galaxies and is subsequently expelled from the host galaxy to the ambient medium. Here we present hydrodynamic simulations investigating the effect of ram-pressure stripping of cluster galaxies. On the other hand the ICM is enriched directly by intra-cluster stars and intra-cluster supernovae. We have also investigated the influence of this mechanism on the chemical enrichment and heating of the ICM. The efficiency, resulting spatial distribution of the metals and the time dependency of this enrichment process on galaxy cluster scale will be shown in this presentation.
\end{abstract}

Baryons in Dark Matter Halos

5-9 October 2004

Novigrad, Croatia

${ }^{*}$ Speaker.

Published by SISSA

http://pos.sissa.it/ 


\section{Introduction}

Clusters of galaxies contain a hot and thin plasma in between the cluster galaxies - the IntraCluster Medium (ICM) which can be observed in X-rays. X-ray spectra of the ICM show metal lines (Fukazawa et. al. 1998 [6]) which indicate that the gas is not only of primordial origin as metals are generally produced in stars. The amount of metals in the ICM is of the same order of magnitude as the amount of metals found in the cluster galaxies. This means that processed material from stars and supernovae must have been ejected into the ICM. Possible enrichment mechanisms of the ICM are the feedback from intra-cluster supernovae (Domainko et al. 2004 [5], Zaritsky et al. 2004 [16], Lin \& Mohr 2004 [11]), feedback from intra-cluster star formation (Gerhard et al 2002 [8]) and material expelled by cluster galaxies. Processes which can remove gas from cluster galaxies are ram-pressure stripping (Gunn \& Gott 1972) [9], galactic winds (De Young 1978 [4]), galaxy-galaxy interactions and jets from active galaxies. Two of these processes (ram-pressure stripping, galaxy-galaxy interactions) are triggered by their surroundings whereas the other two processes (galactic winds and jets) are triggered by violent internal processes.

The effi ciency, time dependences and spatial distributions of these mechanisms are poorly understood. In this contribution we study the effect of ram-pressure stripping and the feedback of intra-cluster supernovae on the chemical evolution of the ICM.

\section{Ram-pressure stripping}

There is now more and more evidence for ram-pressure stripping acting on cluster galaxies. Several examples of ram-pressure effected galaxies in the Virgo cluster can be found (e.g. Vollmer et al. 2004a [14], Vollmer et al. 2004b [15]). These galaxies feature truncated disks and extraplanar cold HI gas.

We study the impact of ram-pressure stripping of cluster galaxies on the ICM. For this purpose we perform combined N-body and hydrodynamic simulations on galaxy cluster scale with an additional semi-numerical model for galaxy formation (van Kampen et al. 1999 [13]). As ram-pressure stripping is an environmental dependent process accurate hydrodynamic treatment is essential. Hydrodynamics is performed with a PPM (Piecewise Parabolic Method: Colella \& Woodward 1984 [2]) which allows to resolve shocks very well. The effect of ram-pressure stripping according to the local properties of the ICM and the properties of the galaxies is included in the calculations. The hydrodynamic simulation is obtained on four nested grids (Ruffert 1992[12]). This technique allows us to cover the cluster center where most of the stripping is expected to happen with high resolution (cell size comparable to galaxy size). Metallicity is used as a tracer to follow the enriched material.

\section{Feedback from intra-cluster supernovae}

Recent observations show that a signifi cant fraction of stars resides in the space between the cluster galaxies (Arnaboldi et al. 2003 [1]). It was also found that a considerable fraction of all supernovae Ia in a galaxy cluster is intergalactic (Gal-Yam et al. 2003 [7]). Those intergalactic supernovae will heat and enrich the surrounding ICM directly (Domainko et al. 2004 [5]).

$074 / 2$ 


\section{First results}

In our simulations we see several subcluster mergers due to hierarchical merger events. Shock waves are the most prominent features in the ICM in connection with merger activity and they can clearly be seen in our simulations. As shock waves are jumps in the density distribution they will clearly influence the effi ciency of the ram-pressure stripping of the cluster galaxies. Therefore we see enhanced mass loss of the galaxies due to ram-pressure stripping during merger events. Another appearing feature is the fact that ram-pressure stripping is getting more efficient when galaxies move closer to the cluster center. This can be understood in terms of increased massloss due to the higher ambient density of the ICM and higher galaxy velocities closer to the cluster center. In principle this creates a metallicity gradient. In addition to that we also see some mixing due to the merger activity. However we still see regions with higher metallicities (see Fig. 1) which are also observed (Hayakawa et al. 2004 [10]). We model observed clusters (like the Coma cluster) to compare simulations with observations. For this purpose we produce surface brightness maps, temperature maps and metallicity maps to compare them directly to the observed maps of the cluster.

In cooling core clusters a central iron excess is observed (De Grandi \& Molendi [3]). Intra-cluster supernovae contribute to produce the iron excess and circumvent the problem of transporting the iron out of the central galaxy into the ICM (Domainko et al. 2004 [5], Lin \& Mohr 2004 [11]).

\section{References}

[1] Arnaboldi, M., Freeman, K. C., Okamura, S., et al. 2003 AJ, 125, 514

[2] Colella, P., Woodward, P. R. 1984, J. Comput. Phys., 54, 174

[3] De Grandi, S. \& Molendi, S. 2001, ApJ, 551, 153

[4] De Young, D. S., 1978, ApJ 223, 47

[5] Domainko, W., Gitti, M., Schindler, S. \& Kapferer 2004 A\&A, 425, L21

[6] Fukazawa, Y. Makishima, K., Tamura, T., et al. 1998, PASJ 50, 187

[7] Gal-Yam, A., Maoz, D., Guhathakurta, P., Filippenko, A. V. 2003, AJ, 125, 1087

[8] Gerhard, O., Arnaboldi, M., Freeman, K. C., Okamura, S. 2002, ApJ, 580, L121

[9] Gunn, J. E., Gott J. R. III, 1972 ApJ, 176, 1

[10] Hayakawa, A., Furusho, T., Yamasaki, N. Y. \& Ohashi, T. 2004, PASJ in press, astro-ph/0409744

[11] Lin, Y., Mohr, J.J., 2004, ApJ in press, astro-ph/0408557

[12] Ruffert, M. 1992, A\&A, 265, 82

[13] van Kampen, E., Jimenez, R., Peacock, J. A. 1999, MNRAS, 310, 43

[14] Vollmer, B., Balkowski, C., Cayatte, V., van Driel, W., Huchtmeier, W. 2004a, A\&A, 419, 35

[15] Vollmer, B., Beck, R., Kenney, J. D. P., van Gorkom, J. H. 2004b, AJ, 127, 3375

[16] Zaritsky, D., Gonzalez, A. H., Zabludoff, A. I. 2004, ApJ, 613, L93 

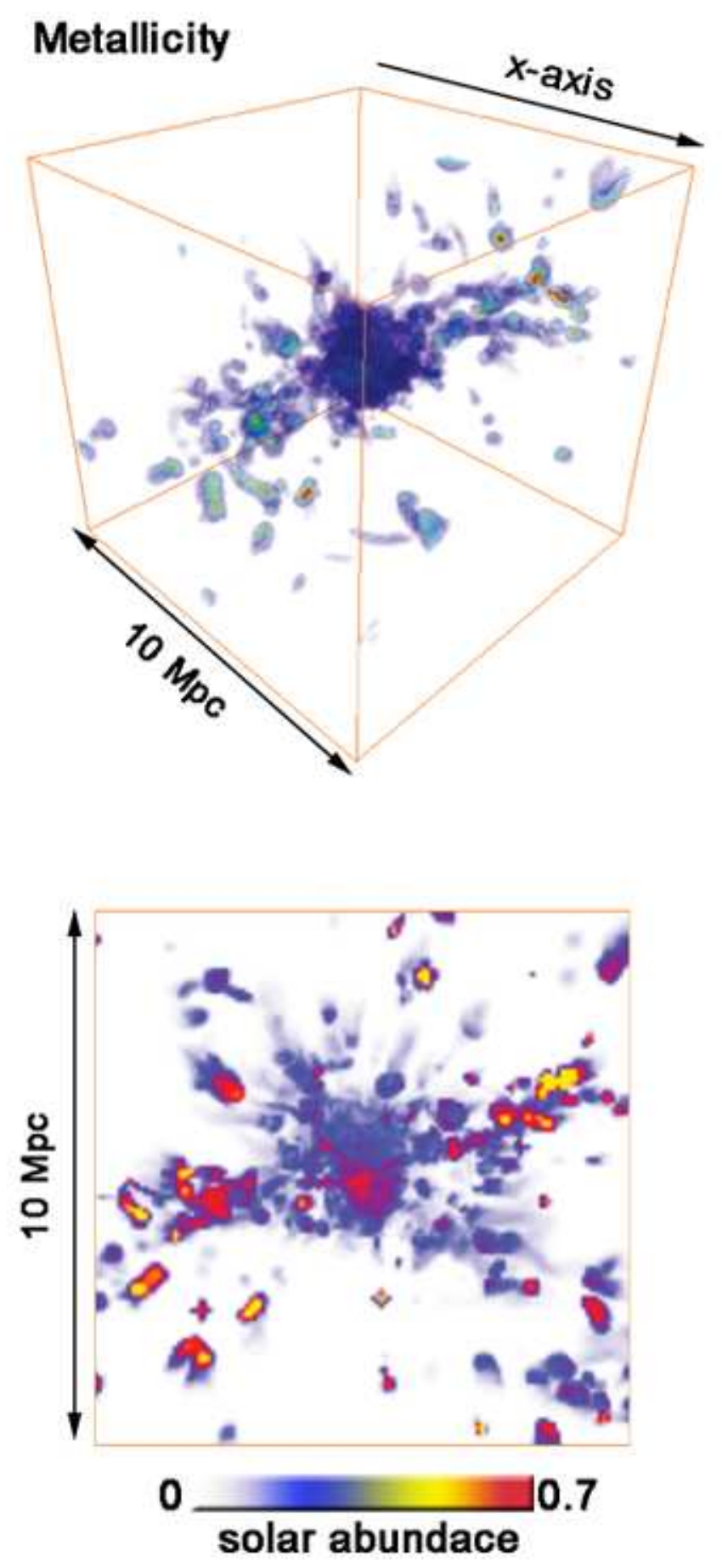

Figure 1: The distribution of stripped, enriched material. The lower image is a projection along the $\mathrm{X}$-axis. Some mixing at the cluster center can be seen. 